Proceedings

\title{
Development of a Novel Platelets Functional Assay Using QCM ${ }^{\dagger}$
}

\author{
Agnieszka E. Strallhofer ${ }^{1}$, Stefan Jung ${ }^{1}$, Christof Jungbauer ${ }^{2}$ and Peter A. Lieberzeit 1,* \\ 1 Department of Physical Chemistry, Faculty for Chemistry, University of Vienna, Währinger Strasse 42, \\ 1090 Vienna, Austria; agnieszka.ewa.strallhofer@univie.ac.at (A.E.S.) \\ 2 Austrian Red Cross, Blood Program, Blood Service for Vienna, Lower Austria and Burgenland, \\ Wiedner Hauptstrasse 32, 1041 Vienna, Austria \\ * Correspondence: peter.lieberzeit@univie.ac.at; Tel.: +43-1-4277-52341; Fax: +43-1-4277-9523 \\ + Presented at the Eurosensors 2017 Conference, Paris, France, 3-6 September 2017.
}

Published: 29 August 2017

\begin{abstract}
Thrombocytes and their metabolites are involved in numerous physiological and pathophysiological processes, such as blood coagulation at the site of ruptured endothelium. Normal platelet aggregation can be suppressed via platelet antagonists or non-steroidal anti-inflammatory drugs (NSAIDs). That's why differentiating between resting ("normal") and inhibited platelets in a quick and precise fashion could be of a great clinical significance. Herein, we present the first step on the way to a novel platelet functionality assay based on Molecularly Imprinted Polymers (MIP) coated onto Quartz Crystal Microbalance (QCM). First results reveal sensor responses that depend on platelet concentration and on incubation of platelets with aspirin: acetyl salicylic acid (AAS).
\end{abstract}

Keywords: molecularly imprinted polymers; MIP; QCM; thrombocytes; acetyl salicylic acid

\section{Introduction and Motivation}

Platelets-also known as Thrombocytes - are a-nucleated blood cells. They are fragments of the cytoplasm of megakaryocytes which differentiate from bone marrow cells and play an important role in hemostasis: by triggering blood coagulation at the site of interrupted endothelium through a mechanism comprising three steps: adhesion, activation and aggregation [1]. Normal platelet function can be suppressed by drugs e.g., per oral intake of either platelet antagonists, or NSAIDs [2]. Furthermore, their functionality can be reduced by clinical conditions, such as Thrombocytopenia and Thrombocytopathie. Because of this, coagulation disorders and their diagnosis are of a great importance in clinical medicine. Although several methods exist to assess platelet functionality, no rapid test is currently available to differentiate between resting "normal" vs. inhibited platelets [3].

Within this work, we present first proof-of-concept results for the development of a new method for assessing the blood coagulation status of thrombocytes, through sensors based on molecularly imprinted polymers (MIP) coated onto the electrodes of quartz crystal microbalances (QCM) [4]. The main difficulty in this case lies in the fact that platelet activation during imprinting has to be avoided, which makes this task more challenging than e.g., erythrocyte MIP [5].

\section{Methods and Results}

Using a two gold-electrode-QCM setup, a thin layer of pre-reacted oligomer (based on urethane) was spin-coated onto both electrodes. The thin film on one electrode was then exposed to a few microliters of a suspension containing $10^{5}$ thrombocytes/ $\mu \mathrm{L}$ in PBS $(\mathrm{pH}=7.4)$ followed by stamping with PDMS layer and drying overnight. The resulting surface was then washed with $0.5 \%$ SDS solution and rinsed with water afterwards, see Figure 1. Figure 2 shows AFM images of the surfaces 
of resulting MIP and corresponding NIP (non-imprinted polymers). While NIP reveals a rather smooth surface, MIP shows cavities with diameter of aprox. 1-3 $\mu \mathrm{m}$, which corresponds to the diameters of thrombocytes.

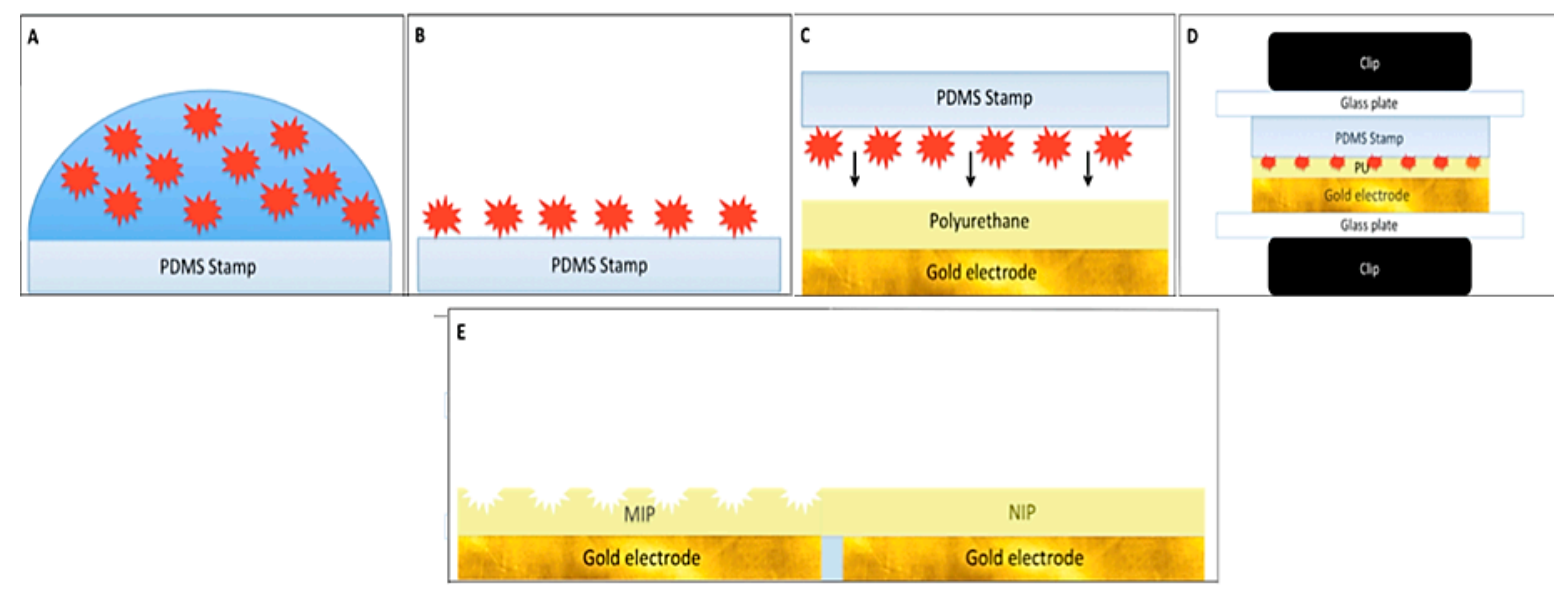

Figure 1. Procedure steps leading to MIP sensors: (A) Thrombocytes in buffer solution adhering onto a PDMS stamp (B) Thrombocytes bind non-covalently to the stamp; (C,D) Template cells are tightly pressed onto a gold electrode (E) Imprints are established, template cells are washed off. One electrode is imprinted with thrombocyte (MIP), while the other is not (NIP).
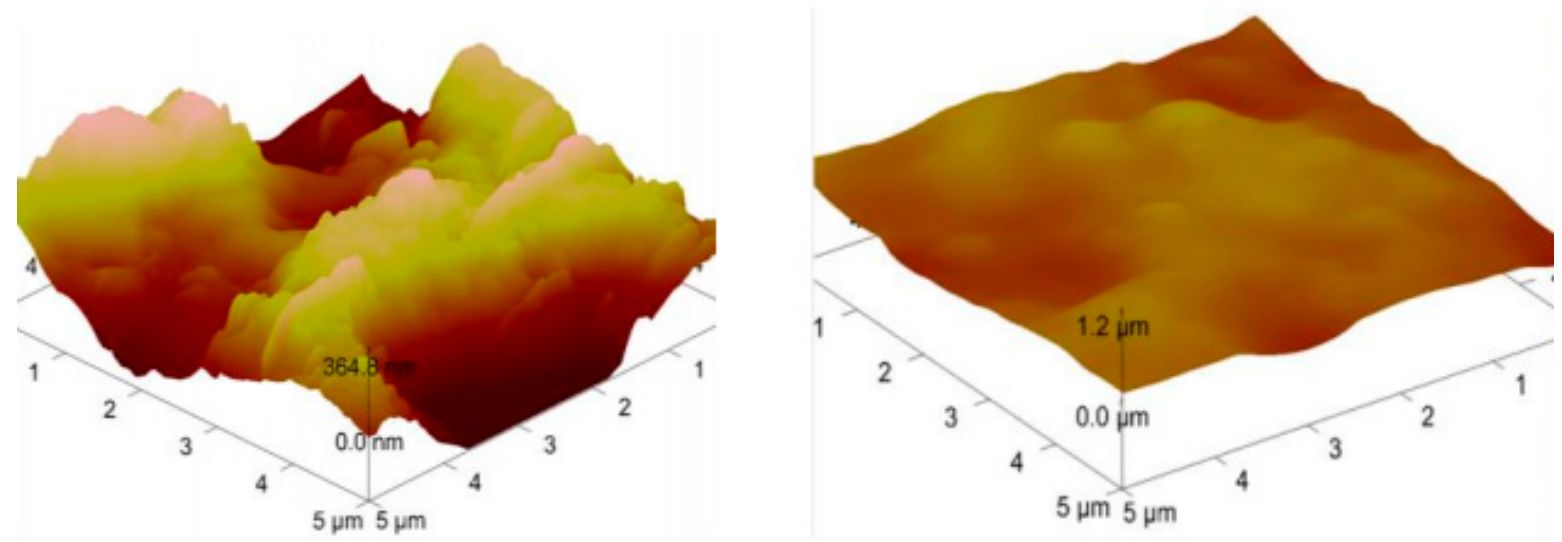

Figure 2. AFM (Atomic Force Microscope) imaging of MIP and NIP after imprinting with platelet rich plasma $(\mathrm{PRP})$ 1:20 dilution $\left(1 \times 10^{5}\right.$ thrombocytes $\left./ \mu \mathrm{L}\right)$.

To validate our setup, we applied different concentrations of platelet rich plasma (PRP), to check: (1) if MIP and NIP show different binding patterns, and (2) to see how different concentrations would affect binding on the electrode surfaces. In Figure 3 one can see that the affinity between platelets and MIP obviously increases with increasing concentration of thrombocytes: "PRP" means "platelet-rich plasma", i.e., blood plasma containing no other cellular components than thrombocytes. Especially during fundamental sensor development, this is easier to handle, than full blood. 


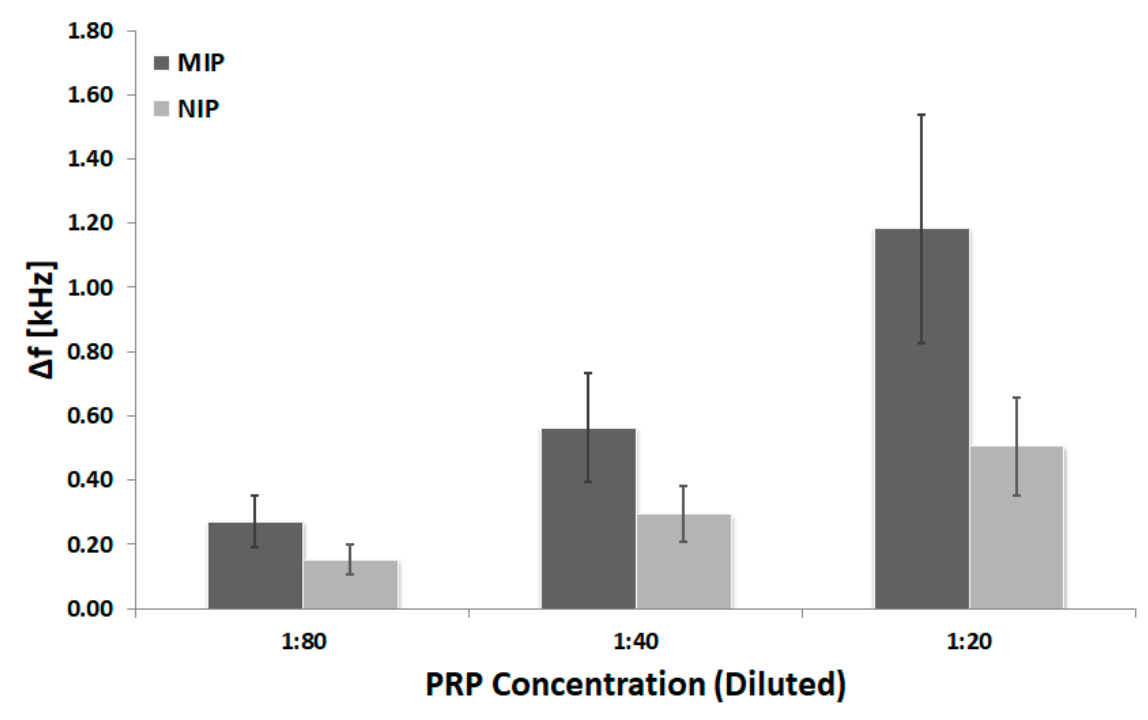

Figure 3. PRP different dilutions vs. corresponding binding response on QCM MIP-NIP electrodes $(n=6) . x$-axis represents different thrombocytes dilutions $\left(1: 80=2.5 \times 10^{4}\right.$ thrombocytes $/ \mu \mathrm{L}$, $1: 40=5 \times 10^{4}$ thrombocytes $/ \mu \mathrm{L}$ and $1: 20=10^{5}$ thrombocytes $\left./ \mu \mathrm{L}\right), \mathrm{y}$-axis represents the binding response on the gold electrode surface on the MIP and NIP sides respectively.

Figure 4 shows preliminary data demonstrating the effects of an important member of the NSAIDs family (Aspirin) on the membrane of thrombocytes. Aspirin inhibits COX-1 and partially COX-2 (intracellular enzymes that modulates important hemostasis mediators) reversibly and non-selectively.That led to substantial change of the signals. Compared to unaltered platelets, those exposed e.g., to $5 \mathrm{mM}$ solutions of acetyl salicylic acid led to sensor responses that are a factor of 2,2 lower. This strongly indicates that inhibition by the antagonist leads to changes in surface chemistry on the cells, which makes them less prone to bind to the surface cavities of the MIP (which were generated by imprinting with active platelets). This comes appreciably in line with previous results obtained for erythrocytes, where MIP turned out selective toward different blood groups [6].

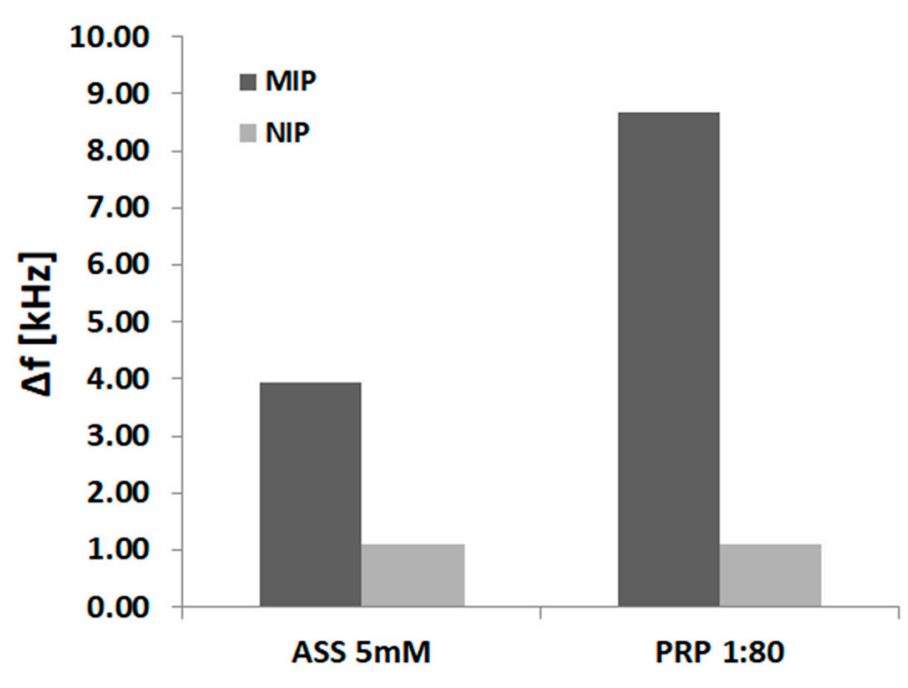

Figure 4. Influence of acetyl salicylic acid on thrombocyte MIP sensor responses.

\section{Conclusions and Future Work}

First of all, we demonstrated successful imprinting with dormant thrombocytes with full ability to coagulate. The resulting MIP indeed incorporate those cells, which leads to increased frequency signal. Furthermore, exposing thrombocytes to aspirin indirectly obviously changes their surface 
structure by modulating the membrane receptors and their relevant pathways. Hence we showed for the first time a proof of concept that it possible to detect these surface medications using a real-time, fast and reliable tool. Our QCP-MIP, a real-time analytical setup, in principle enables us to differentiate thrombocytes in their physiological conditions (untreated, non-inhibited) from thrombocytes that are pre-treated with a commonly used clinical NSAID (aspirin). This opens up potential applications in many medical fields, such as a quick reliable tool for early diagnostics regard the platelets pathophysiology, without the need for sophisticated, costly routine laboratory procedures.

Conflicts of Interest: The authors declare no conflict of interest.

\section{References}

1. Golebiewska, E.M.; Poole, A.W. Platelet secretion: From haemostasis to wound healing and beyond. Blood Rev. 2015, 29, 153-162.

2. Schafer, A.I. Effects of nonsteroidal anti-inflammatory drugs on platelet function and systemic hemostasis. J. Clin. Pharmacol. 1995, 35, 209-219.

3. Israels, S.J. Laboratory testing for platelet function disorders. Int. J. Lab. Hematol. 2015, 37 (Suppl. 1), 18-24, doi:10.1111/ijlh.12346.

4. Kutner, C. Artificial Biosensors: How Can Molecular Imprinting Mimic Bio-recognition? Trends Biotechnol. 2016, 34, 922-941, doi:10.1016/j.tibtech.2016.05.011.

5. Seifner, A.; Lieberzeit, P.; Jungbauer, C.; Dickert, F.L. Synthetic receptors for selectively detecting erythrocyte ABO subgroups. Anal. Chim. Acta 2009, 651, 215-219, doi:10.1016/j.aca.2009.08.021.

6. Hayden, O.; Mann, K.J.; Krassnig, S.; Dickert, F.L. Biomimetic ABO blood-group typing. Angew. Chem. Int. Ed. Engl. 2006, 45, 2626-2629.

(C) 2017 by the authors. Licensee MDPI, Basel, Switzerland. This article is an open access article distributed under the terms and conditions of the Creative Commons Attribution (CC BY) license (http://creativecommons.org/licenses/by/4.0/). 